\title{
INFLAÇÃO INERCIAL SOB MUDANÇAS DE REGIME: ANÁLISE A PARTIR DE UM MODELO MS-ARFIMA, 1944-2009
}

\author{
Erik Alencar de Figueiredo* \\ André M. Marques ${ }^{\dagger}$
}

\begin{abstract}
Resumo
Este artigo analisa a dinâmica da inflação brasileira a partir de uma estrutura fracionária com mudança de regime markoviana, MS-ARFIMA, fornecida por Tsay \& W. (2009). A vantagem dessa metodologia em relação a outras abordagens é a estimação simultânea das prováveis mudanças de regime e do coeficiente fracionário, $d$, que expressa a memória de longo prazo (inércia) da inflação brasileira. Os principais resultados sugerem a vigência de dois regimes distintos, sendo que o regime de baixa inflação é o mais persistente. Conclui-se também que a memória de longo prazo da inflação é sensível a mudanças de regime
\end{abstract}

Palavras-chave: Inflação Inercial; Dependência de Longo Prazo; MS-ARFIMA.

\begin{abstract}
The main goal of this paper is search for the long run dependence in the Brazilian inflation rate allowing regime switching by the MS-ARFIMA model. The principal contribution of this paper is the simultaneous and consistent estimation of long memory coefficient $d$ and the several regimes that encompass them. The results enable us to conclude that there were two regimes in the Brazilian economy with high persistence: hyperinflation regime and low inflation regime. The long run memory of inflation rate is dependent upon regime switching. The low inflation regime is the more persistent one.
\end{abstract}

Palavras-chave: Inertial Inflation; MS-ARFIMA; Long Run Dependence. JEL classification: C22; E31.

\footnotetext{
* Programa de Pós-graduação em Economia. Universidade Federal da Paraíba. E-mail: eafigueiredo@gmail.com

† Programa de Pós-graduação em Economia. Universidade Federal do Rio Grande do Norte. Email: andremmarques@yahoo.com.br
} 


\section{Introdução}

O sucesso da política de estabilização de uma economia depende crucialmente do diagnóstico correto acerca da natureza da inflação vigente. Por isso, a explicação para a evolução do índice geral de preços de uma economia constitui preocupação central não apenas para os teóricos, mas especialmente para os formuladores da política macroeconômica dos países.

A despeito do histórico de alta inflação, mormemente no período de sua industrialização e nos anos 1980, desde a implantação do Plano Real, ${ }^{1}$ há cerca de quinze anos, a economia brasileira vem experimentando uma taxa de inflação anual sob controle quando comparada com seus patamares históricos. ${ }^{2} \mathrm{O}$ período de estabilidade se dá a partir da remoção da memória do processo inflacionário (inércia) no Brasil provocando a desindexação da economia, mediante uma Reforma Monetária precedida de indexação plena. Para os teóricos da inflação inercial, "se fosse possível suprimir a memória inflacionária [indexação formal e informal], as (...) condições objetivas da economia manteriam os preços muito próximos da estabilidade" (Resende (1985b): 131).

Contudo, não obstante o sucesso do Plano Real, muitos preços ainda continuam sendo regulados por contratos indexados, que pouco respondem às condições de oferta e demanda da economia. Desse modo, é plausível supor que uma parte importante de inércia (memória) tenha sido preservada mesmo com as sucessivas mudanças de regime provocadas pelos diferentes planos de estabilização, incluindo o Plano Real.

Por ser uma regra formal, a indexação preservada institucionalmente pelo governo em diversos setores da economia como saúde, energia e comunicações, pode ter impedido a eliminação plena da memória inflacionária. ${ }^{3}$ Além disso, por ser fomentada explicitamente pelo governo, é uma regra que pode incentivar comportamentos similares em outros setores da economia.

Diversos estudos corroboram a hipótese da inflação inercial no Brasil. Inicialmente, as abordagens basearam-se nos testes para raiz unitária. Os procedimentos são diversos e envolvem a inclusão de mudanças na parte determinística do processo gerador da série ou sua robustez frente a observações discrepantes. Neste grupo de estudos, destacam-se Cati et al. (1999), Campêlo \& Cribari-Neto (2003) e Cribari-Neto \& Cassiano (2005).

O procedimento empírico é simples: considera-se que a inflação é integrada de ordem $d, I(d)$. Caso $d=1, I(1)$, a série será não estacionária, exibindo inércia. Se $d=0, I(0)$, os choques serão estacionários e não haverá memória inflacionária. A principal limitação dessa abordagem é que ela considera apenas a indexação plena, $I(1)$, o que constitui um caso particular. De uma forma geral, o comportamento inercial é caracterizado por valores de $d>0$ e não só para $d=1$. Neste sentido, os modelos fracionários, em especial os modelos Auto-regressivos Fracionários de Médias Móveis, $\operatorname{ARFIMA}(p, d, q)$, constituem alternativas mais flexíveis e permitem a captação de comportamentos inerciais diversos, posto que $d \in(0,1)$.

\footnotetext{
${ }^{1}$ Formulado e implantado pelos teóricos da inflação inercial na economia brasileira em meados de 1994. Os fundamentos teóricos mais importantes acerca da hipótese da inflação inercial podem ser encontrados especialmente em Lopes \& Williamson (1980), Modiano (1983), Modiano (1985), Resende (1985a) Resende (1985b) e Lopes (1985).

${ }^{2}$ Ver Simonsen (1985) para um histórico da inflação brasileira.

${ }^{3}$ Ver Figueiredo \& Ferreira (2002) para uma descrição detalhada deste tópico.
} 
Ademais, os testes de raiz unitária são, em geral, não-robustos frente à caracterização fracionária. Diebold \& Rudebush (1991), por exemplo, mostram que os testes para raiz unitária de Dickey-Fuller apresentam um baixo poder em face dessa característica. De forma mais específica, Lee \& Amsler (1997) mostram que as abordagens tradicionais não conseguem distinguir entre um comportamento não estacionário, porém reversível à média $(0,5<d<1) \mathrm{e}$ uma raiz unitária $(d=1)$. Diante disso, a caracterização das séries de tempo a partir dos modelos ARFIMA, introduzidos independentemente por Granger \& Joyeux (1980) e Hosking (1981), têm sido utilizados de forma muito frequente na literatura.

Utilizando o modelo ARFIMA e diversos estimadores para o parâmetro de integração, $d$, Reisen et al. (2003) apontam para um comportamento fracionário da série da inflação brasileira. Em resumo, os coeficientes estimados sugerem que a inflação é caracterizada pela longa memória, ou seja, não estacionária porém reversível à média $(0,50<d<1)$. Este comportamento é compatível com a existência de inércia, porém, sem a indexação plena.

Os resultados de Reisen et al. (2003) constituem um importante referencial para o estudo da dinâmica inflacionária brasileira. Porém, eles negligenciam uma característica marcante na evolução dos índices, qual seja: os diferentes regimes ou patamares de inflação. Comparando-se o período pós-Plano Real com a década de 1980, por exemplo, é possível identificar um regime de alta inflação e outro de baixa inflação em que o ponto de mudança parece situar-se em torno do Plano Real.

Diante disso, a detecção de um possível comportamento fracionário para as séries deve considerar a hipótese de mudanças de regime. Em caso contrário, como mostrado por Diebold \& Inoue (2001), Bos et al. (1999) e Granger \& Hyung (2004), a existência de mudanças de regime pode gerar padrões espúrios de dependência similares a processos de memória longa, mesmo quando cada regime é um processo de memória curta. ${ }^{4}$

A literatura aponta algumas soluções para essa questão. Bos et al. (1999) propõem um modelo baseado em determinações exógenas dos pontos das quebras. A limitação do estudo de Bos et al. (1999) é a escolha dos pontos das quebras fixadas exogenamente pelo próprio pesquisador, que requer um considerável conjunto de informações a priori. Baillie \& Morana (2009) sugerem um modelo mais complexo, considerando a existência de quebra estrutural na média e na variância do processo gerador dos dados. ${ }^{5}$ Tsay \& W. (2009) propõem uma abordagem alternativa estimando endogenamente os pontos de quebra, que resulta em uma combinação do modelo ARFIMA com o MarkovSwitching (MS-ARFIMA). Este último modelo apresenta um comportamento satisfatório diante de uma amostra finita e pode ser estimado a partir de critérios iterativos.

A principal justificativa para inclusão simultânea de mudanças de regime e memória longa é que esse ferramental permite identificar corretamente se os padrões de memória longa observados nos dados são efetivamente compatíveis com processos do tipo ARFIMA ou são gerados de forma espúria pelas mudanças de regime.

\footnotetext{
${ }^{4}$ Para uma análise empírica desta possibilidade para dados brasileiros, ver Laurini \& Portugal (2004).

${ }^{5}$ Essa classe de modelos tem se mostrado robusta frente à caracterização de séries financeiras. Ver Baillie et al. (1996) e Baillie et al. (2002).
} 
Isso posto, o objetivo deste artigo é investigar a memória de longo prazo da inflação brasileira estimando-se endogenamente as prováveis mudanças de regime, que refletem as alterações de política econômica em cada período. Para alcançar esse objetivo estima-se um modelo MS-ARFIMA, que é capaz de captar simultaneamente a mudança de regime e a presença de memória longa em altas defasagens de um processo auto-regressivo, representada pelo coeficiente fracionário $d$. Esse coeficiente reflete o grau de indexação da economia medido no longo prazo.

Os principais resultados alcançados no trabalho indicam a vigência de pelo menos dois regimes distintos e altamente persistentes: um regime de hiperinflação com um grau de indexação acima da unidade, e outro, de baixa inflação, com um grau de indexação abaixo da unidade. Conclui-se que para o regime de estabilidade de preços a inflação brasileira exibe um comportamento não estacionário, porém reversível à média. Além disso, constatou-se que o regime de baixa inflação pode ser interpretado como uma característica quasepermanente da economia brasileira.

Por fim, o artigo está organizado do seguinte modo: na segunda seção, é apresentado o modelo ARFIMA, a ser estimado como uma base preliminar; na terceira seção é apresentado o modelo MS-ARFIMA; na seção quatro é feita a descrição da base de dados e são discutidos os principais resultados do estudo. Por fim, seção cinco, são feitos os comentários finais.

\section{Modelo ARFIMA}

O modelo $\operatorname{ARFIMA}(p, d, q)$ discreto é definido por

$$
\Phi(B)(1-B)^{d} X_{t}=\Theta(B) \epsilon_{t}, \quad \epsilon_{t} \sim R B\left(0, \sigma_{\epsilon}^{2}\right),
$$

onde $t=1,2, \ldots, n, B$ é um operador de defasagens, $\Phi(B)=1-\phi_{1} B-\ldots-\phi_{p} B^{p}$ e $\Theta(B)=1-\theta_{1} B-\ldots-\theta_{q} B^{q}$ são polinômios de ordens $p$ e $q$, respectivamente, com todas as raízes fora do círculo unitário e $R B$ representa um ruído branco. $\mathrm{O}$ parâmetro $d$ mensura a possível longa memória da série, que será estacionária e invertível caso $d \in(-0.5,0.5)$.

A literatura aponta uma série de métodos para estimação do coeficiente fracionario. ${ }^{6}$ Entretanto, este estudo opta por duas ferramentas distintas. Ambas baseiam-se em procedimentos semi-paramétricos, partindo da seguinte densidade espectral para (1):

$$
f(w)=f_{u}(w)\{2 \sin (w / 2)\}^{-2 d}, \quad w \in[-\pi, \pi],
$$

onde $f_{u}(\cdot)$ é a densidade espectral para o processo $\operatorname{ARMA}(p, q)$. Aplicando logaritmo na expressão (2) e adicionando os termos $\ln f_{u}(0)$ e $\ln \left\{I\left(\omega_{j}\right)\right\}$ obtémse:

$$
\ln I\left(\omega_{j}\right)=\ln f_{u}(0)-d \ln \left(2 \sin \left(\omega_{j} / 2\right)\right)^{2}+\ln \left\{I\left(\omega_{j}\right) / f\left(\omega_{j}\right)\right\}+\ln \left\{f_{u}\left(\omega_{j}\right) / f_{u}(0)\right\} .
$$

Uma vez que $\lim _{n \rightarrow \infty} \frac{g(n)}{n}=0$ o último termo de (3) se torna desprezível quando comparado aos demais, pois os valores estão concentrados muito próximo de zero. Daí pode-se usar a aproximação dada por

\footnotetext{
${ }^{6}$ Para detalhes, ver Beran (1994).
} 


$$
\ln I\left(\omega_{j}\right) \approx \ln f_{u}(0)-d \ln \left(2 \sin \left(\omega_{j} / 2\right)\right)^{2}+\ln \left\{I\left(\omega_{j}\right) / f\left(\omega_{j}\right)\right\} .
$$

Ou seja, a equação acima pode ser representada por uma regressão simples na forma: $\ln y_{j}=\theta+\gamma x_{j}+u_{j}$. Onde $y_{j}=I\left(\omega_{j}\right), \theta=\ln f_{u}(0), x_{j}=\left(2 \sin \left(\omega_{j} / 2\right)\right)^{2}$ e $u_{j}=\ln \left\{I\left(\omega_{j}\right) / f\left(\omega_{j}\right)\right\}$. Diante disso, Geweke \& Porter-Hudak (1983) propõem um estimador de Mínimos Quadrados para o parâmetro de longa memória:

$$
\hat{\gamma}=\frac{\sum\left(x_{j}-\bar{x}\right) y_{j}}{\sum\left(\left(x_{j}-\bar{x}\right)^{2}\right.} .
$$

Sendo $\hat{d}_{p}=-\hat{\gamma}$. O domínio dessas funções é restrito à janela $g(n)=n^{\alpha}, \quad 0 \leq$ $\alpha \leq 1$.

O segundo estimador, $\hat{d}_{s p}$, introduzido por Reisen (1994), substitui $I\left(\omega_{j}\right)$ na expressao (4), por um periodograma calculado com autocovariância amostral suavizada pela janela de Parzen. Neste caso, a janela $g(n)$ é escolhida sobre um ponto de trucagem definido por $m=n^{\beta}$, $\operatorname{com} 0<\beta<1$.

O método de Geweke \& Porter-Hudak (1983) tem sido largamente utilizado na literatura. ${ }^{7}$ Já Reisen (1994) propõe o uso da função periodograma suavizado, que é um estimador consistente da função espectral. ${ }^{8}$ Grosso modo, a diferença fundamental entre os estimadores $\hat{d}_{p}$ e $\hat{d}_{s p}$, é que no segundo há um alisamento preliminar do periodograma, usada para inferir (2).

\section{Modelo MS-ARFIMA}

Defina $\left\{s_{t}\right\}_{t=1}^{T}$ como as possíveis trajetórias para os $N$-estados da cadeia de Markov. Considere que a variável latente $s_{t}$ assume, em cada ponto do tempo, apenas valores discretos, $s_{t}=1,2, \ldots, N$, com a seguinte matriz de transição de probabilidade:

$$
\mathcal{P} \equiv\left(\begin{array}{cccc}
p_{11} & p_{21} & \cdots & p_{N 1} \\
p_{12} & p_{22} & \cdots & p_{N 2} \\
\vdots & \vdots & \ddots & \vdots \\
p_{1 N} & p_{2 N} & \cdots & p_{N N}
\end{array}\right)
$$

onde $p_{i j}=\operatorname{Pr}\left(s_{t}=j \mid s_{t-1}=i\right)$ e $\sum_{j=1}^{N} p_{i j}=1, \forall i$.

Isto posto, quando a taxa de inflação, $Y_{t}$, segue um MS-ARFIMA, então

$$
Y_{t}=\mu_{s_{t}} I\{t \geq 1\}+(1-B)^{-d_{s_{t}}} \sigma_{s_{t}} Z_{t} I\{t \geq 1\}=\mu_{s_{t}} I\{t \geq 1\}+v_{t},
$$

onde $Y_{t}$ é a taxa de inflação observada no tempo $t, I\{\cdot\}$ é uma função indicadora e $Z_{t}$ é um processo ARMA com média zero. A função $I\{\cdot\}$ controla a influência do passado infinito de $Z_{t}$ sobre $Y_{t}$, dado que o $d_{s_{t}} \geq 0.5$. Supõe-se ainda que a variável latente $s_{t}$ é independente de $Z_{t}$ para todo $t$ (suposição A de Tsay \& W. (2009)).

Caso $N=1$, (3.1) torna-se um ARFIMA padrão. Isto posto, pode-se representar a função densidade condicionada (cdf) para $U_{t}$ por $f\left(Y_{t} \mid \mathcal{S}_{t}, \mathcal{Y}_{t-1} ; \xi\right)$. Onde $\mathcal{S}_{t}$ é um vetor de estados, $\mathcal{Y}_{t-1}$ é um vetor de variáveis defasadas e $\xi$

\footnotetext{
${ }^{7}$ Destacando-se, Diebold \& Rudebush (1989) e Baillie et al. (1996) , entre outros.

${ }^{8}$ O método de Reisen (1994) também tem sido bastante utilizado. Ver Chen et al. (1994) e Reisen et al. (2003), entre outros.
} 
um vetor contendo os parâmetros e as transições de probabilidade do modelo. A função de verossimilhança resultante é solucionada a partir de suposições sobre os valores iniciais, distribuição e especificação para $Z_{t}$. Para tanto, Tsay \& W. (2009) sugerem a adoção do algoritmo de Viterbi (1967), pois os algoritmos normalmente utilizados na solução dos problemas markovianos, como, por exemplo, (Hamilton (1989) e Hamilton (1990)), não podem ser empregados na estrutura (3.1).

O algoritmo proposto de Tsay \& W. (2009) parte da seguinte função de verossimilhança para (3.1):

$$
L\left(\mathcal{S}_{t}, \mathcal{Y}_{t} ; \xi\right)=\prod_{t=1}^{T}(2 \pi)^{-1 / 2}\left(v_{t-1}\right)^{-1 / 2} \exp \left\{-\frac{\left(y_{t}-\hat{y}_{t}\right)^{2}}{2 v_{t-1}}\right\} \operatorname{Pr}\left(s_{t} \mid s_{t-1}\right),
$$

onde $\hat{y}_{t}$ corresponde à previsão um passo à frente para $y_{t}, v_{t-1}$ é a previsão um passo a frente para a variância e $\operatorname{Pr}\left(s_{t} \mid s_{t-1}\right)$ é a probabilidade markoviana já definida anteriormente.

A grande dificuldade associada a (3.2) é que não se conhece a trajetória da variável latente $s_{t}$. Diante disso, adota-se uma modificação no procedimento de Viterbi (1967), onde, estipulam-se $N^{T}$ possíveis rotas para a série de inflação. Em sua versão original, o algoritmo de Viterbi soluciona a função de verossimilhança a partir de uma programação dinâmica em tempo discreto, com um número de estados finito e com a parte aleatória seguindo um ruído branco. A modificação proposta por Tsay \& W. (2009) considera a mesma estrutura, porém, com ruídos ARFIMA. Para tanto, é necessário especificar valores iniciais para os parâmetros do modelo em cada regime, quais sejam: média, probabilidades de transição, parâmetro fracionário, variância e parâmetros de memória curta. ${ }^{9}$

Em suma, o modelo MS-ARFIMA reune duas importantes características das séries econômicas e financeiras: o comportamento fracionário e a mudança de regime markoviana. A seleção endógena dos pontos das quebras e a facilidade de implementação a partir de critérios iterativos são um atrativo para a adoção desta estrutura. Além do mais, as simulações de Tsay \& W. (2009) demonstram o bom desempenho destes modelos em amostras finitas.

\section{Resultados}

Nesta seção serão apresentados os dados e os principais resultados do estudo. O objetivo é determinar o nível de persistência e, por conseguinte, de inércia da série de inflação. Serão adotados dois procedimentos. No primeiro, utilizar-se-ão estruturas ARFIMA convencionais. Os resultados alcançados nesta etapa não trarão novas evidências para a literatura posto que tal estratégia já foi implementada por Reisen et al. (2003).

Ciente da necessidade de se considerar possíveis mudanças de regime, o estudo adotará o modelo que une a integração fracionária e a mudança de regime markoviana (MS-ARFIMA). Nesta segunda etapa serão discutidas, além do nível de persistência, as características relacionadas a cada regime, como a probabilidade de ocorrência, volatilidade da inflação, entre outras.

\footnotetext{
${ }^{9}$ Os detalhes relacionados ao algoritmo podem ser obtidos nas seções 2 e 3 de Tsay \& W. (2009). O procedimento computacional encontra-se em: http://idv.sinica.edu.tw/wjtsay/pdf/ms-arfima.un.
} 


\subsection{Dados e Resultados Preliminares}

A inflação brasileira é representada pela variação percentual do Índice Geral de Preços - Disponibilidade Interna (IGP-DI), calculado pela Fundação Getúlio Vargas. Os dados foram obtidos junto ao endereço eletrônico do Instituto de Pesquisa Econômica Aplicada - IPEA. ${ }^{10}$ Foram consideradas as observações mensais compreendidas entre fevereiro de 1944 e agosto de 2009. A evolução da série pode ser observada na Figura 1.

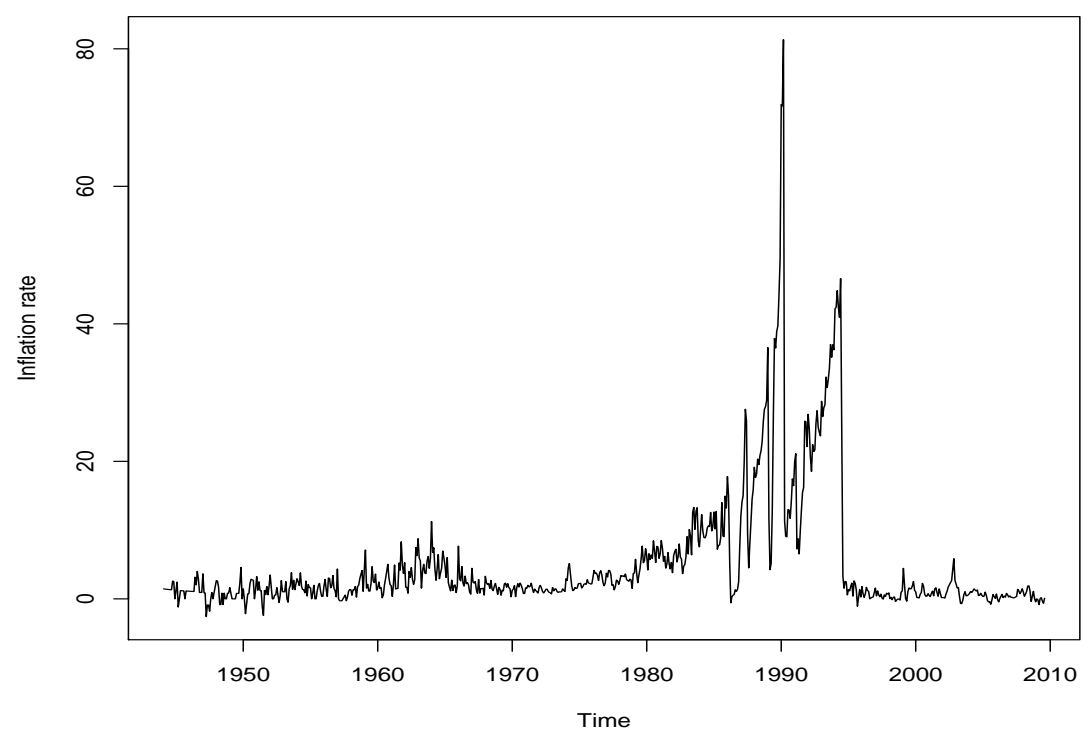

Figura 1: Evolução da Inflação Brasileira: 1944:02-2009:08.

A inspeção visual sugere a presença de outliers, quebras estruturais, regimes diferenciados, entre outros. Todas estas características já foram discutidas, em maior ou menor escala, pela literatura especializada. Cati et al. (1999) e Campêlo \& Cribari-Neto (2003), destacam que o período compreendido entre 1986 e 1992 possui, além das características mencionadas, fortes influências de inliers, ou seja, planos de estabilização que atuam de forma transitória sobre a trajetória de longo prazo da série.

A possível mudança de regime foi discutida, para o período pós-Plano Real, por Laurini \& Vieira (2005). Os autores detectaram, a partir de um modelo espaço-estados com erros GARCH, que a inércia inflacionária é mais acentuada em períodos de crise econômica, destacando-se a crise cambial em 1999 e a incerteza decorrente da eleição presidencial em 2002. De acordo com os autores, este comportamento explica a aceleração inflacionária em momentos de instabilidade.

Em suma, a observação da Figura 1, somada às evidências contidas na literatura, não permitem tratar a dinâmica inflacionária brasileira a partir de um modelo com coeficientes fixos ao longo do tempo, tampouco não robus-

\footnotetext{
${ }^{10}$ http://www.ipeadata.gov.br.
} 
tos frente a mudanças bruscas na parte determinística do processo gerador da série.

A observação do correlograma (Figura 2), sugere um comportamento nãoestacionário e possivelmente caracterizado por longa memória, face o lento decaimento das autocorrelações amostrais. Diante disso, aplicam-se testes visando captar um possível componente fracionário da série. A Tabela 1 apresenta os principais resultados para o parâmetro de longa memória da inflação brasileira, usando os métodos propostos por Geweke \& Porter-Hudak (1983) - GPH(1983) - e Reisen (1994). Considerou-se o bandwidth $\alpha=0,50$ e um ponto de trucagem, na estimativa de Reisen (1994), $\beta=0,90$. Assim como em Reisen et al. (2003), incluiram-se os resultados específicos do subperíodo de janeiro de 1944 a dezembro de 1985. Este procedimento visa observar a sensibilidade dos resultados frente aos inliers.

Series igpdi.ts

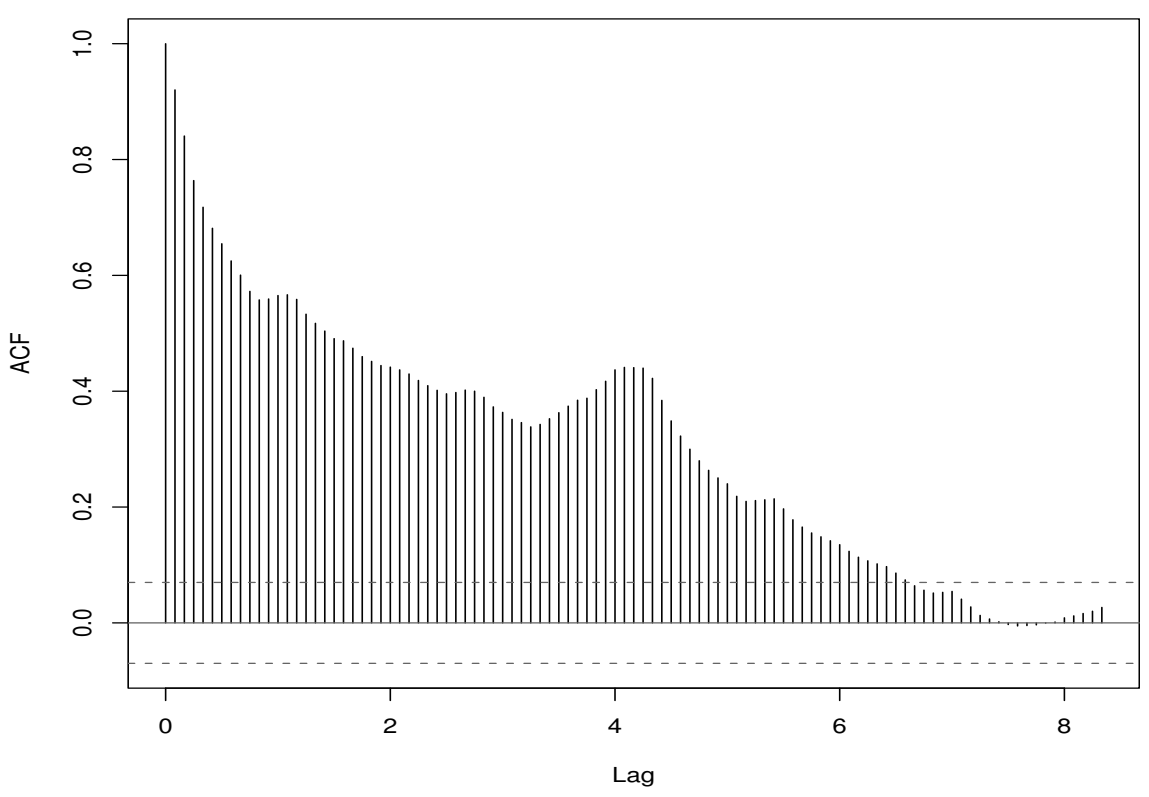

Figura 2: Função de autocorrelação para a inflação brasileira. O eixo x representa os lags em anos.

Tabela 1: Estimativa do coeficiente de longa memória (d) na inflação brasileira

\begin{tabular}{ccccc}
\hline Amostra & $\hat{d}_{p}$ & D.P. & $\hat{d}_{s p}$ & D.P. \\
\hline $1944: 02-2009: 08$ & 0,8152 & 0,1462 & 0,7228 & 0,0599 \\
$1944: 02-1985: 12$ & 0,9364 & 0,1773 & 0,8603 & 0,0570 \\
\hline
\end{tabular}

De uma forma geral, os resultados da Tabela 1 sugerem que a inflação brasileira apresenta memória longa. Os parâmetros fracionários, situados entre 0.72 e 0.93 , são condizentes com um comportamento não estacionário, porém reversível à média. As estimativas foram próximas às obtidas por Baillie et al. (1996) e Reisen et al. (2003). A caracterização em dois períodos indica que 
os inliers não possuem influência sobre o parâmetro de longa memória. Caso ela ocorresse, a estimativa para toda a amostra apontaria um comportamento fracionário, já o período livre de inliers se caracterizaria por uma raiz unitária.

Como já mencionado, estes resultados não trazem novidade em relação a literatura existente. Contudo, eles servem como base para um importante questionamento: as estimativas para os parâmetros fracionários da inflação brasileira sofrem influência de possíveis mudanças de regime?

Para Diebold \& Inoue (2001) e Granger \& Hyung (2004), a omissão das quebras estruturais viesam as estimativas do parâmetro fracionário. Sendo assim, torna-se pertinente adotar uma estrutura fracionária com mudança de regime.

\subsection{Resultados a Partir do Modelo MS-ARFIMA}

A hipótese central desta seção é que a inflação brasileira segue um modelo MS-ARFIMA $(1, d, 1)$, com dois estados, i.e.: ${ }^{11}$

$$
\begin{gathered}
Y_{t}=\mu_{s_{t}} I\{t \geq 1\}+(1-B)^{-d_{s_{t}}} \sigma_{s_{t}} Z_{t} I\{t \geq 1\} \\
\left(1-\phi_{1} B\right) Z_{t}=\left(1-\theta_{1} B\right) \epsilon_{t} .
\end{gathered}
$$

Os resultados para as estimações de (4.1) encontram-se na Tabela 2. Foram consideradas três especificações alternativas. Utilizou-se o algoritmo desenvolvido por Tsay \& W. (2009), também utilizado em Tsay \& W. (2009). O processo de inferência envolve a otimização a partir do algoritmo quasi-Newton BFGS. ${ }^{12}$ Foram consideradas até 100 replicações.

Tabela 2: Estimativas para os parâmetros do modelo MS-ARFIMA

\begin{tabular}{lcccccc}
\hline & \multicolumn{2}{c}{ ARFIMA $(1, d, 1)$} & \multicolumn{2}{c}{$\operatorname{ARFIMA}(1, d, 0)$} & \multicolumn{2}{c}{$\operatorname{ARFIMA}(0, d, 1)$} \\
\hline & Estimativa & D.P. & Estimativa & D.P. & Estimativa & D.P. \\
\hline$d_{1}$ & 1,0868 & 0,0478 & 0,9907 & 0,0141 & 1,0180 & 0,0545 \\
$d_{2}$ & 0,8442 & 0,0696 & 0,8354 & 0,0542 & 0,8564 & 0,0781 \\
$p_{11}$ & 0,9594 & 0,0158 & 0,9072 & 0,0263 & 0,9577 & 0,0153 \\
$p_{22}$ & 0,9908 & 0,0037 & 0,9837 & 0,0049 & 0,9890 & 0,0042 \\
$\sigma_{1}$ & 5,5918 & 0,3228 & 5,6967 & 0,8676 & 5,4017 & 0,2971 \\
$\sigma_{2}$ & 1,0229 & 0,0288 & 1,3791 & 0,8086 & 0,9753 & 0,0279 \\
$\mu_{1}$ & 6,4763 & 3,2326 & 6,5117 & 3,1654 & 7,0922 & 3,9696 \\
$\mu_{2}$ & 1,8765 & 0,7531 & 1,0197 & 0,7654 & 1,6781 & 0,6327 \\
$\phi_{1}$ & 0,5457 & 0,0676 & 0,4728 & 0,0498 & - & - \\
$\theta_{1}$ & $-0,8299$ & 0,0397 & - & - & 0,4785 & 0,6070 \\
\hline AIC & 230,44 & $-242,47$ & & $-1,8490$ \\
$L^{*}$ & $-1,8445$ & $-1,8586$ & \\
\hline \multicolumn{7}{c}{234} \\
\hline
\end{tabular}

A partir dos resultados mostrados na Tabela 2, constata-se que todos os parâmetros estimados são estatisticamente significativos a $1 \%$ e que seus valores indicam a vigência de dois regimes distintos para a economia brasileira. Além

\footnotetext{
${ }^{11}$ Tanto $\phi_{1}$, quanto $\theta_{1}$ (ou ambos) podem ser iguais a zero. Convén ressaltar que, assim como em Tsay \& W. (2009), a adoção de dois regimes não se baseou em um critério estatístico para seleção de modelos.

${ }^{12}$ Processo de otimização não-linear de Broyden-Fletcher-Goldfarb-Shanno. Para maiores detalhes, ver Tsay \& W. (2009).
} 
disso, observa-se que dentre as três especificações avaliadas, o modelo que melhor descreve o comportamento da inflação brasileira é o MS-ARFIMA $(1, d, 1)$, em face do valor do critério de informação de Akaike (AIC).

Para o caso do primeiro regime tem-se uma economia com indexação plena com uma trajetória explosiva para a taxa de inflação. Neste regime o patamar médio de inflação mensal é muito elevado e a volatilidade da inflação também é cerca de cinco vezes maior do que no regime de baixa inflação. Esses três aspectos constituem as características predominantes do primeiro regime.

A evolução no tempo e a alternância entre os regimes podem ser visualizados na Figura 3, onde são mostrados os diferentes regimes que caracterizam o comportamento da inflação no Brasil, a partir da estrutura MS$\operatorname{ARFIMA}(1, d, 1)$. A Figura 4 demonstra a evolução do coeficiente fracionário ao longo do tempo. Nota-se que a predominância do regime de alta inflação é característica do período da industrialização do País e da década de 1980 até o início dos anos 1990.

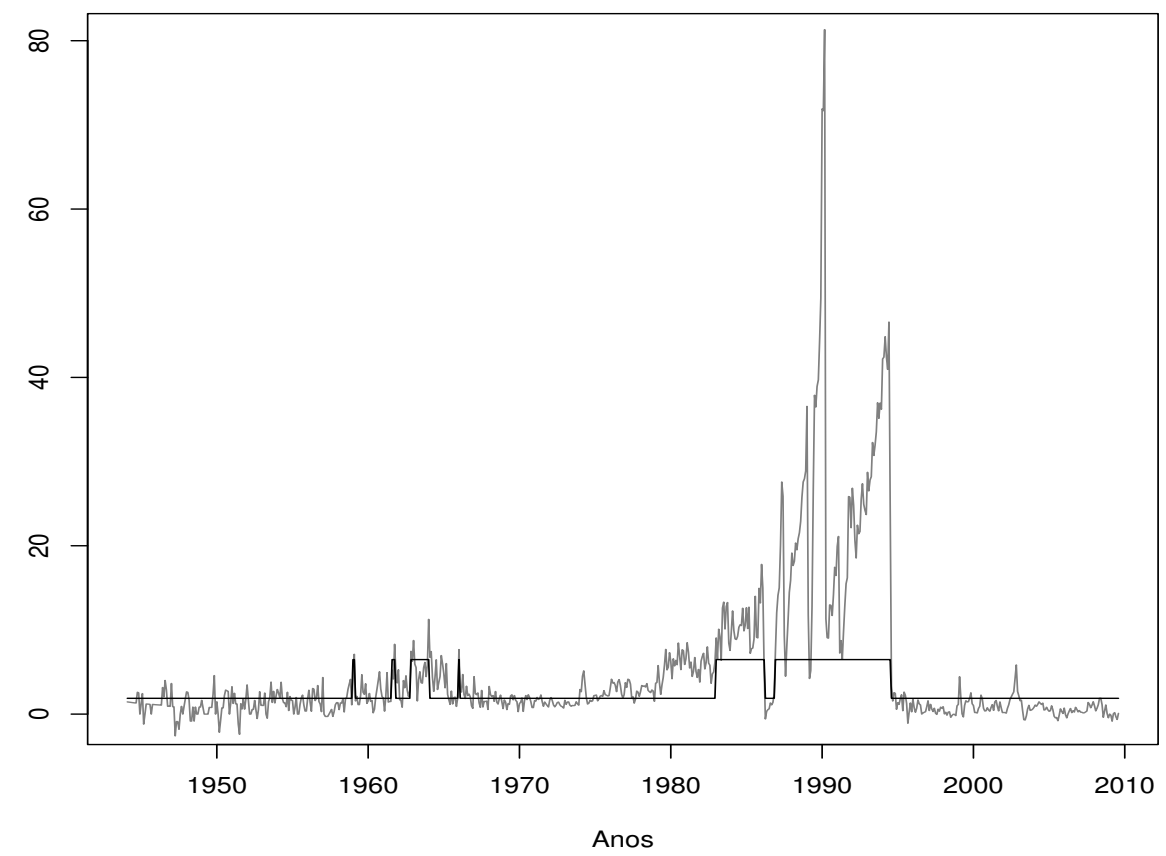

Figura 3: Evolução dos regimes markovianos para inflação brasileira.

Para o caso do segundo regime tem-se uma economia com memória de longo prazo abaixo da unidade, indicando a natureza acumulativa, porém não mais explosiva, do processo inflacionário. Neste regime o patamar médio de inflação mensal é muito baixo, quando comparado com o primeiro regime, e a baixa volatilidade da inflação é também uma característica predominante. Esses três aspectos constituem as características mais importantes do segundo regime. Como se pode observar na Figura 3, esse regime foi predominante no início da industrialização, nos anos 1970 até o início dos anos 1980 e após o Plano Real.

Quando se observa as probabilidades associadas a cada regime, na Tabela 2 , nota-se que embora ambos os regimes sejam de alta persistência, é precisamente o regime de baixa inflação, baixa volatilidade e média mensal que 


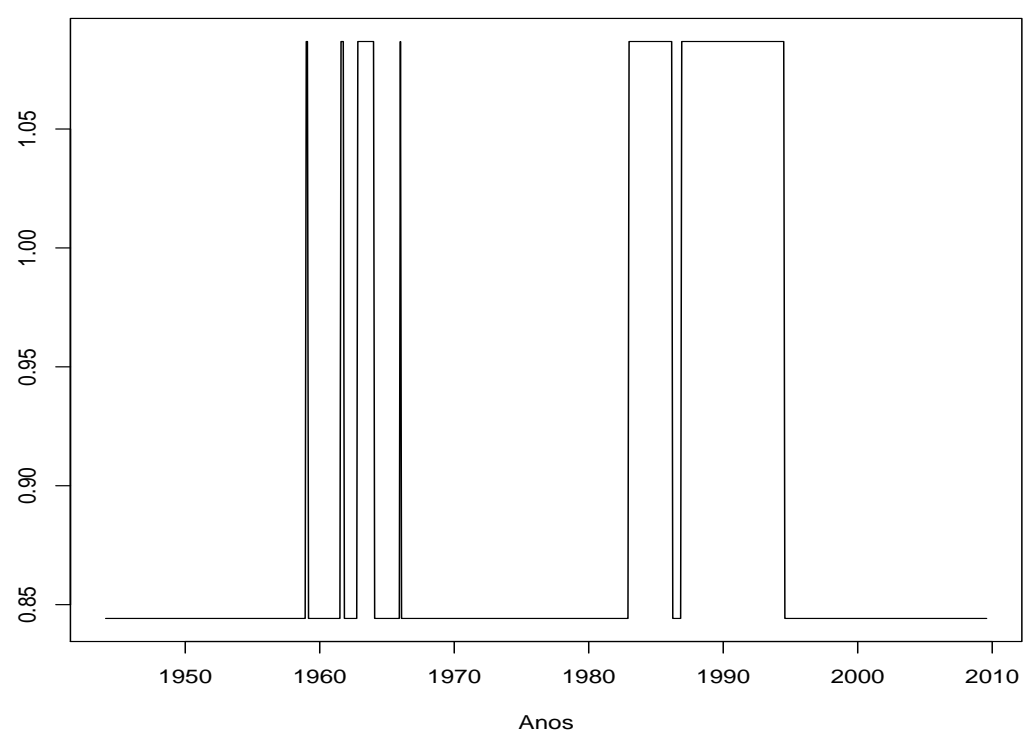

Figura 4: Evolução do parâmetro fracionário da inflação brasileira.

constitui o regime mais persistente na economia brasileira. Como sua probabilidade associada é de 0,9908 pode-se dizer que a estabilidade de preços que vigora na economia brasileira apresenta um caráter quase-permanente.

Em outros termos, o regime de baixa inflação que vigora atualmente na economia brasileira apresenta um grau de persistência muito elevado. Este resultado pode ser interpretado como um indicador da solidez do processo de estabilização da economia brasileira e é consistente com o que se esperava teoricamente por ocasião da implantação do Plano Real. ${ }^{13}$

Ainda com relação aos resultados expostos na Tabela 2, quando são comparados os valores dos coeficientes de memória longa (inércia) para ambos os regimes (hiperinflação e baixa inflação) e, tendo em conta outros trabalhos na área, como o de Reisen et al. (2003) e Baillie et al. (1996), há indícios que a omissão das mudanças de regime para o caso da inflação brasileira pode ter provocado a subestimação do coeficiente de memória longa da inflação. As estimações feitas por esses autores captaram apenas parcialmente o grau de indexação de longo prazo da economia brasileira.

Reisen et al. (2003), por exemplo, estimaram o coeficiente de memória longa para a inflação brasileira utilizando a mesma medida de inflação e obtiveram $\hat{d}_{s p}=0,705$ e $\hat{d}_{s}=0,753$, para o período 1944:02 - 2000:02. Como não foi constatada nenhuma mudança de regime após 2000:02, a diferença entre os resultados pode ser proveniente da ausência de uma caracterização explícita para as mudanças de regime nas estimações dos autores.

Em síntese, pode-se dizer que os resultados indicam a sensibilidade do grau de indexação da economia consoante o regime vigente. Isto é, para períodos de baixa inflação e baixa volatilidade como o atual, a memória de longo prazo da inflação, representado pelo coeficiente fracionário $d$, também se al-

\footnotetext{
${ }^{13}$ Ver Bacha (1997).
} 
tera substancialmente de um valor acima da unidade para um valor abaixo da unidade, indicando que a natureza explosiva da inflação brasileira é parte apenas do regime que vigorou predominantemente na década de 1960 e 1980.

\section{Considerações Finais}

O objetivo principal do estudo foi investigar a memória de longo prazo da inflação brasileira, medida pela variação percentual mensal do IGP-DI, estimandose o coeficiente fracionário $d$, tendo em conta explicitamente as mudanças de regime ao longo do período 1944-2009.

A partir da estimação de um modelo ARFIMA e do modelo MS-ARFIMA, constatou-se que o valor do coeficiente fracionário tende a ser subestimado quando as mudanças de regime são omitidas. Ao longo do período analisado pelo menos dois regimes podem ser caracterizados na economia brasileira. $\mathrm{O}$ primeiro, de hiperinflação, constitui um regime de indexação plena, em que o coeficiente de memória longa é maior do que a unidade. Na ausência de intervenção política a inflação brasileira assumiria uma trajetória explosiva (divergente) no longo prazo.

Os sucessivos planos de estabilização, que visavam à desindexação da economia, alteraram o grau de indexação da economia para um valor abaixo da unidade, tornando a inflação brasileira não estacionária, porém, convergente para a média no longo prazo. Isso constitui o segundo regime detectado nas estimações, com uma média e volatilidade substancialmente menores que no regime de hiperinflação. As maiores alterações provocadas pelas políticas econômicas entre os dois regimes situam-se na redução do grau de indexação da economia, no patamar médio mensal e na volatilidade da inflação.

No regime de baixa inflação, apesar de o processo ser reversível à média, em virtude de sua dependência de longo prazo a diluição de eventuais choques sobre a taxa de inflação corrente requer longos períodos de tempo. Embora as observações passadas estejam separadas por altas defasagens da inflação corrente, elas estão fortemente correlacionadas. Por isso, pode-se dizer que a inflação corrente carrega uma memória de longo prazo (inércia), representada pelo coeficiente fracionário $d$.

Apesar de ambos os regimes apresentarem alta persistência, expressa pelas suas probabilidades associadas, constatou-se que o regime de baixa inflação tem uma probabilidade associada próxima da unidade, o que pode ser interpretado como um regime de caráter quase-permanente. Este resultado pode ser interpretado como um indicador de solidez da estabilidade de preços que vigora na economia brasileira nos últimos quinze anos.

Quanto à manutenção do atual regime, os resultados sugerem que uma estratégia eficaz consiste menos na geração de choques negativos de demanda do que na revisão e extinção gradual de antigos mecanismos que ainda restam na economia que cumprem o papel de carregar para o futuro a memória de um passado de alta inflação.

Em um trabalho futuro sugere-se que o algoritmo de otimização seja expandido, a fim de incluir a possibilidade de se captar um número superior de regimes (incluindo o regime de hiperinflação, por exemplo). Tal procedimento permitiria um melhor ajuste no período compreendido entre 19801994. Outro avanço seria a adoção de uma estrutura ARCH-GARCH para os resíduos, algo que não foi possível dentro dos limites deste trabalho. 


\section{Agradecimentos}

O primeiro autor gostaria de agradecer o suporte financeiro do $\mathrm{CNPq}$, por meio do Projeto 475225/2009-0. Estamos em débito com dois pareceristas anônimos e com os Editores Walter Belluzzo Junior e Irineu de Carvalho Filho.

\section{Referências Bibliográficas}

Bacha, E. (1997), O Plano Real: uma avaliação., in 'O Brasil pós-Real: a política econômica em debate.', Campinas: Unicamp-IE.

Baillie, R., Chung, C. \& Tieslau, M. (1996), 'Analysing inflation by the fractionally integrated ARFIMA-GARCH model.', Journal of Applied Econometrics, 11, 23-40.

Baillie, R., Han, Y. \& Kwo, N. (2002), 'Further long memory properties of inflationary shocks.', Southern Economic Journal 68, 496-510.

Baillie, R. \& Morana, C. (2009), 'Investigating inflation dynamics and structural change with an adaptive ARFIMA approach', Working Paper Series: International Centre for Economic Research .

Beran, J. (1994), Statistics for Long-Memory Processes, New York: Chapman and Hall.

Bos, C., Franses, P. \& Ooms, M. (1999), Long memory and level shifts: Reanalyzing inflation rates, Empirical Economics.

Campêlo, A. K. \& Cribari-Neto, F. (2003), 'Inflation Inertia and Inliers: The Case of Brazil', Revista Brasileira de Economia 54, 713-739.

Cati, R. C., Garcia, M. G. P. \& Perron, P. (1999), 'Unit Roots in the Presence of Abrupt Governmental Interventions with Application to Brazilian Data', Journal of Applied Econometrics 14, 27-56.

Chen, G., Bovas, A. \& Peires, S. (1994), 'Lag window estimation of the degree of differencing in fractionally integrated Time Serie models.', Journal of Time Series Analysis 15, 473-487.

Cribari-Neto, F. \& Cassiano, K. (2005), 'Uma análise da dinâmica inflacionária brasileira.', Revista Brasileira de Economia 59, 535-566.

Diebold, F. \& Inoue, A. (2001), 'Long memory and regime switching.' , Journal of Econometrics 105, 131-59.

Diebold, F. X. \& Rudebush, G. D. (1989), 'Long memory and persistence in aggregate output', Journal of Monetary Economics 24, 189-209.

Diebold, F. X. \& Rudebush, G. D. (1991), 'On the power of Dickey-Fuller tests against fractional alternatives', Economic Letters 35, 155-160.

Figueiredo, F. M. R. \& Ferreira, T. P. (2002), Os preços administrados e a inflação no Brasil, Trabalhos para Discussão 59, Banco Central do Brasil.

Geweke, J. \& Porter-Hudak, S. (1983), 'The estimation and applications of the long memory time series modesls', Journal of Time Series Analysis 4(4). 
Granger, C. \& Hyung, N. (2004), 'Occasional structural breaks and long memory with an application to the S\&P500 absolute stock returns', Journal of Empirical Finance 11(3), 399-421.

Granger, C. W. J. \& Joyeux, R. (1980), 'An introduction to long-memory time series models and fractional differencing', Journal of Time Series Analysis $1,15-29$.

Hamilton, J. D. (1989), 'A New Approach to the Economic Analysis of Nonstationary Time Series and the Business Cycle', Econometrica 57, 357-384.

Hamilton, J. D. (1990), 'Analysis of time series subject to changes in regime', Journal of Econometrics 45(1-2), 39-70.

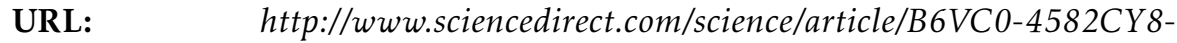
20/2/af4ef1535da31d69fad61731d08faf45

Hosking, J. R. M. (1981), 'Fractional Differencing', Biometrika 68, 165-176.

Laurini, M. \& Portugal, M. (2004), 'Long memory in the R/US exchange rate: a robust analysis', Brazilian Review of Econometrics 24(1), 109-147.

Laurini, M. \& Vieira, H. (2005), 'A dynamic econometric model for inflationary inertia in Brazil', IBMEC, no prelo .

Lee, H. \& Amsler, C. (1997), 'Consistency of the KPSS unt root test against fractionally integrated alternative', Economic Letters 55(2), 151-160.

Lopes, F. (1985), 'Inflação inercial, hiperinflação e desinflação: notas e conjecturas', Revista de Economia Política 5, 135-151.

Lopes, F. \& Williamson, J. (1980), 'A teoria da inflação consistente', Estudos Econômicos 10(1), 61-99.

Modiano, E. (1983), 'A dinâmica de salários e preços na economia brasileira: 1966/ 81', Pesquisa e Planejamento Econômico 13(1), 39-68.

Modiano, E. (1985), 'Salários, preços e câmbio: os multiplicadores dos choques numa economia indexada', Pesquisa e Planejamento Econômico 15(1), 132.

Reisen, V. (1994), 'Estimation of the fractional difference parameter in the ARIMA(p,d,q) model using the smoothed periodogram', Journal of Time Series Analysis 15, 335-350.

Reisen, V., F., C.-N. \& Jensen, M. (2003), 'Long memory inflationary dynamics: the case of Brazil', Studies in Nonlinear Dynamics and Econometrics 7, 1157-1173.

Resende, A. (1985a), 'A moeda indexada: nem mágica nem panacéia.', Revista de Economia Política 5, 124-129.

Resende, A. (1985b), 'A moeda indexada: uma proposta para eliminar a inflação inercial.', Revista de Economia Política 5, 130-134.

Simonsen, M. (1985), 'A inflação brasileira: lições e perspectivas.', Revista de Economia Política 5, 15-30. 
Tsay, W. \& W., H. (2009), 'A generalized ARFIMA process with MarkovSwitching fractional differencing parameter.', Journal of Statistical Computation AND Simulation 79, 731-745.

Viterbi, A. (1967), 'Error bounds for convolutional codes and an asymptotic optimum decoding algorithm.', IEEE Transactions on Signal Processing, IT 13, 260-269. 